\title{
DTA Study on Oxidation of Lithium Soap Grease
}

\author{
Seiichirō HIRONAKA, Masayuki TAKAHASHI*, and \\ Toshio SAKURAI** \\ Tokyo Institute of Technology (2-12-1, Ookayama, Meguro-ku, Tokyo)
}

\begin{abstract}
The phase behavior of lithium soap/hydrocarbon oil and lithium soap/synthetic oil greases has been investigated using DTA. The change in the shape and size of soap micelle in the grease was examined with an electron microscope. It has been found that the phase behavior and the shape and size of the soap micelles in the grease are greatly affected by the oxidation stability of the base oil used. For the lithium stearate/squalane grease, the phase transition temperature and the $\mathrm{L} / \mathrm{D}$ value of the fibers formed by the soap micelles decreased with the oxidation time. However those of the grease prepared with pentaerythritol tetraoctanoate which was stable for oxidation were hardly affected. These facts may be rcduced to a small amount of the polar compounds produced by the oxidation of a base oil.
\end{abstract}

\section{Introduction}

The phase transitions in lubricating grease have been investigated in detail by $\operatorname{Vold},{ }^{1,},{ }^{21}$ Suggitt, ${ }^{3)}$ and $\operatorname{Cox}^{4)}$ and other authors utilizing DTA. The phase transition of lithium soap grease depends mainly upon the properties of the soap micelles in the grease. $\mathrm{Cox}^{5}$ and $\mathrm{Uzu}^{6}$ ) have suggested that the phase diagrams are affected by the molecular weight of the hydrocarbon as a base oil.

Recently, the authors have studied the effect of impurities on the phase behavior and the change in the shape and size of soap micelles with the phase transition in a lithium soap/ hydrocarbon oil system $^{7}$. It has been found that the phase diagrams are affected by the impurities existing in the grease, such as oxidation products of hydrocarbon oil which are produced during the preparation of the grease and unreacted free acid.

In the present study, the lithium soap greases with different type of base oils were prepared in nitrogen atmosphere using hydrocarbon oil and synthetic oil as the base oils, and the effect of oxidation on the phase behavior of the grease was investigated by DTA and electron

* Present address; Ajinomoto Co., Ltd. (1-1, Suzuki -cho, Kawasaki-ku, Kawasaki)

** Present address; Tokai University, c/o The Japan Petroleum Institute (Nisseki Bldg., 2-4, 3-Chome, Marunouchi, Chiyoda-ku, Tokyo) microscopy.

\section{Experimental}

Commercially available high-purity grade stearic acid was purified by repeated recrystal= lization. Lithium stearate was prepared from high purity lithium hydroxide and stearic acid. To remove unreacted stearic acid, lithium stearate was extracted by benzene and finally dried.

Squalane, a synthetic product, was purified by distillation. Pentaerythritol tetraoctanoate was prepared by an esterification procedure. Amberlyst-15 was used as the catalyst and toluene as azeotropic solvent. To obtain high purity tetraester, the reaction mixture containing partial-esters was further reacted with octanoyl chloride. The ester was purified by distillation. The remaining polar compounds as contaminants were removed from the ester by percolation through alumina and Florisil columns.

Squalane and pentaerythritol tetraoctanoate were used as the base oils (Table-1). The greases were prepared by mixing lithium stea= rate and the base oil, heating to $210 \sim 230^{\circ} \mathrm{C}$ under constant stirring for $10 \mathrm{~min}$, and then allowing the hot mixture to cool down to room temperature in nitrogen atmosphere.

The oxidation test of greases was pursued in a thermostated chamber at $95^{\circ} \mathrm{C}$ in air. The phase behaviors of the lithium soap greases 
Table-1 Properties of base oils.

\begin{tabular}{l|c|c|c|c|c|c}
\hline \multicolumn{1}{c|}{ Base oil } & $\begin{array}{c}\text { Saponification value } \\
\mathrm{mg} \mathrm{KOH} / \mathrm{g}\end{array}$ & $\begin{array}{c}\text { Acid value } \\
\mathrm{mg} \mathrm{KOH} / \mathrm{g}\end{array}$ & $\begin{array}{c}\text { Density } \\
d_{20^{20}}\end{array}$ & $\begin{array}{c}\text { Refractive Index } \\
n_{\mathrm{D}}^{20}\end{array}$ & \multicolumn{2}{|c}{ Viscosity (cSt) } \\
\hline Squalane & - & - & 0.811 & 1.4535 & 20.40 & 4.17 \\
$\begin{array}{l}\text { Pentaerythritol } \\
\text { tetraoctanoate }\end{array}$ & $351\left(351^{\circ}\right)$ & $<0.1$ & 0.965 & 1.4511 & 27.47 & 5.35 \\
\hline
\end{tabular}

*calculated value

were studied with the DT-20 B type DTA apparatus, Shimadzu Ltd. The apparatus con= stant was determined by the use of stearic acid, benzoic acid, and tin as the standard materials. The heat of phase transition was calculated from the relation of apparatus constant against temperature. The shape and size of soap micelles in the greases were examined with the JEM-SS type electron microscope, JEOL Co., Ltd.

\section{Results and Discussion}

The change of the phase behavior of $20 \%$ by weight lithium stearate/squalane grease with oxidation time is shown in Fig. -1 . The first prime phase transition temperature (l'st P.T.T.) was observed for the unoxidized grease and the peak in DTA diagram due to the transition decreased with the oxidation time. On the other hand, the first transition temperature was not observed before the oxidation and observed after $3 \mathrm{~h}$. These behaviors corresponded to the

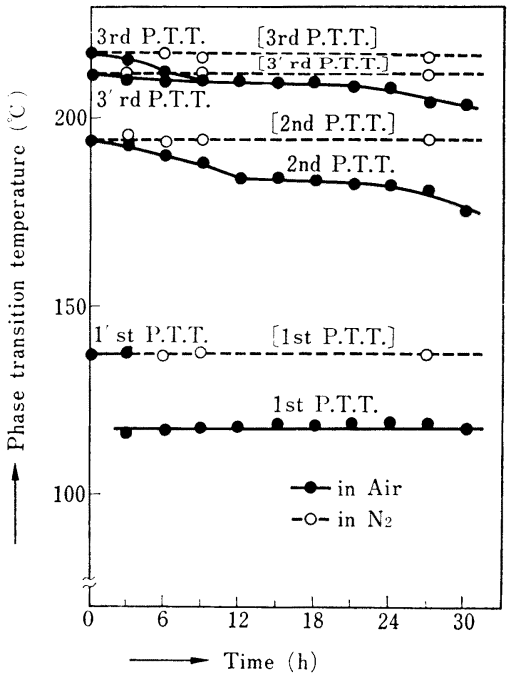

Fig.-1 Effect of oxidation on the phase behavior of lithium stearate/squalane grease (Soap: 20 wt $\%$ ), oxidation temperature $: 95^{\circ} \mathrm{C}$. changes in the first prime and first transition temperatures by the addition of the polar compounds such as fatty acids and aliphatic alcohols.")

The second transition temperature decreased gradually with the oxidation time. The two close transition temperatures, the third and third prime phase transition temperatures, approached each other with the oxidation time and finally became indistinguishable, and then decreased with the oxidation time. These facts coincided well with the effect of the addition of the polar compounds. Therefore, it is reasonable to conclude that the experimental results are due to the effect of the polar compounds as the oxidation products of the base oil.

The oxidation of squalane was confirmed from the characteristic absorption of carbonyl group by IR. All the transition temperatures of the grease prepared with the oxidized squa= lane were also lower compared with those of the unoxidized squalane grease. This also confirms that the oxidation products of the base oil affect the phase behavior of the grease.

On the other hand, the phase behavior of the grease after the deterioration test at $95^{\circ} \mathrm{C}$ in nitrogen atmosphere did not change. This fact may confirm the effect of the oxidation on the phase behavior of the grease.

Fig. -2 shows the effect of the oxidation on the phase behavior of lithium stearate/penta= erythritol tetraoctanoate grease. The grease prepared with pentaerythritol tetraoctanoate was not oxidized because the base oil was more stable for oxidation and its phase behavior hardly changed. The first transition tempera $=$ ture of the pentaerythritol tetraoctanoate base grease showed lower value compared with the first prime transition temperature of the squalane base grease before the oxidation and was much the same with the first transition temperature observed after the oxidation. The third prime 
transition temperature was not observed for the pentaerythritol tetraoctanoate base grease. These facts may be reduced to the polarity of pentaerythritol tetraoctanoate.

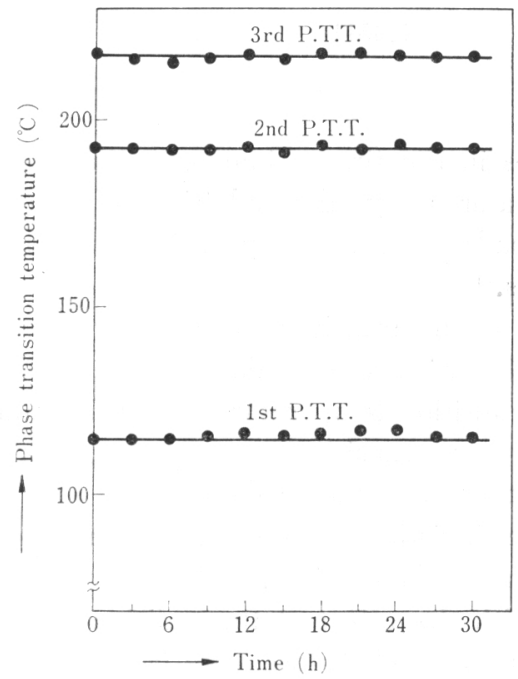

Fig.-2 Effect of oxidation on the phase behavior of lithium stearate/pentaerythritol tetra= octanoate grease (Soap : $20 \mathrm{wt} \%$ ), oxidation temperature : $95^{\circ} \mathrm{C}$.
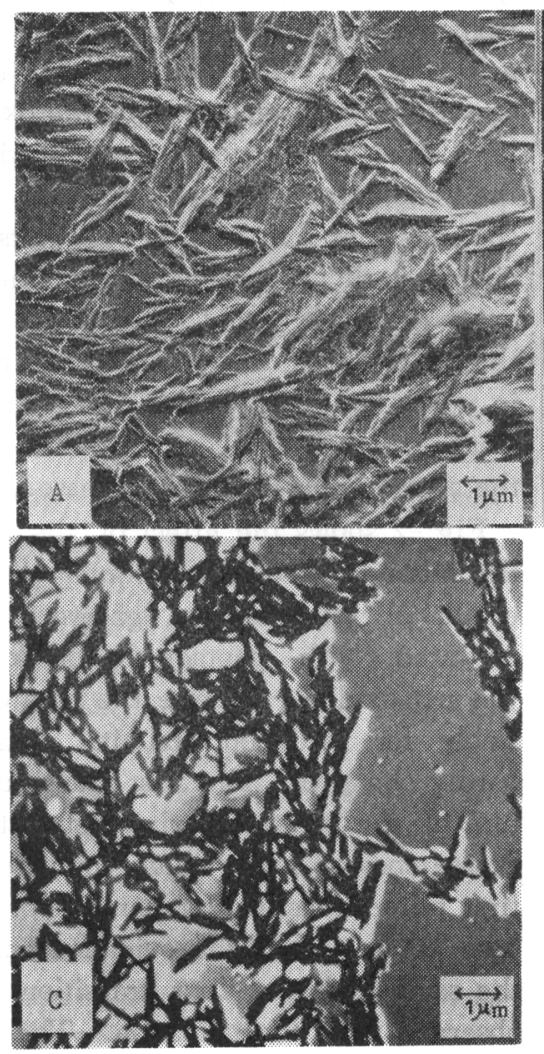

The effect of oxidation on the calculated heat of phase transition of lithium stearate/squalane grease is shown in Fig.-3. The heat of the first prime phase transition decreased sharply

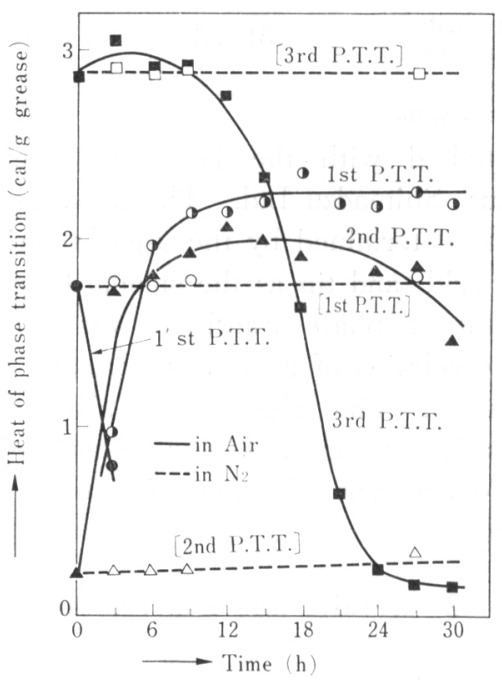

Fig.-3 Effect of oxdation on the calculated heat of phase transition of lithium stearate/squa= lane grease (Soap : $20 \mathrm{wt} \%$ ), oxidation temperature $95^{\circ} \mathrm{C}$.

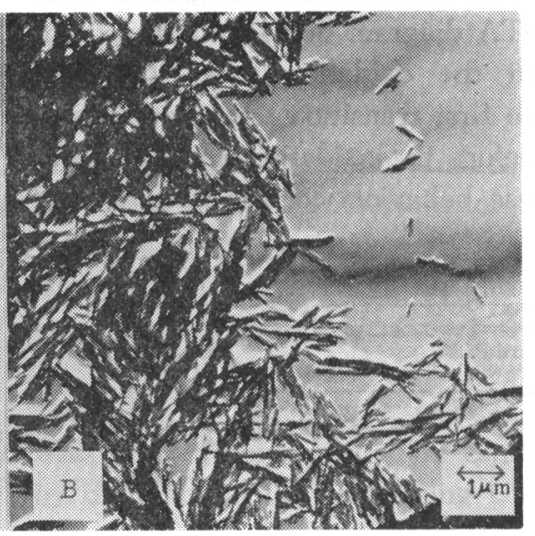

Fig.-4 Electron micrograph of lithium stearate/squalane grease.

A : Oxidation time $0 \mathrm{~h}$

B : " $6 \mathrm{~h}$

C : " $30 \mathrm{~h}$

Oxidation temperature $: 95^{\circ} \mathrm{C}$.

In air. 

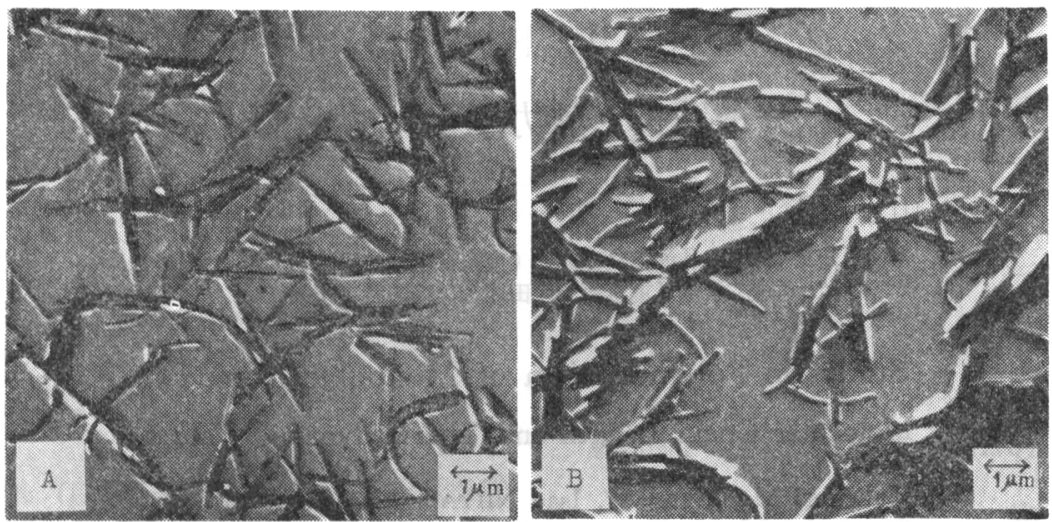

Fig.-5 Electron micrograph of lithium stearate/pentaerythritol tetraoctanoate grease.

A : Oxidation time $0 \mathrm{~h}$

B : $" 30 \mathrm{~h}$

Oxidation temperature $: 95^{\circ} \mathrm{C}$, In air.

with the oxidation time and finally diminished, while that of the first phase transition appeared and increased with the oxidation of the grease, and finally reached to the definite value. The heat of the second phase transition changed almost similarly to that of the first phase tran= sition. The heat of the third phase transition decreased remarkably with the oxidation time. Thus, these results confirm the significant effect of the oxidation on the phase behavior of the grease.

From the above results, it may be suggested that the phase behavior of the grease depends significantly on the oxidation stability of the base oil as well as the molecular weight of he base oil shown by $\mathrm{Cox}^{5}$ and $\mathrm{Uzu}^{6}$ ) and the impurities such as organic polar compounds. ${ }^{\text {?) }}$

Electron micrographs of the lithium soap mi= celles in lithium stearate/squalane and lithium stearate/pentaerythritol tetraoctanoate greases are shown in Fig. -4 and 5, respectively. In the squalane base grease, the soap micelles formed fibers matted together before the oxidation, but $\mathrm{L} / \mathrm{D}$ value and size of the mat of the fibers decreased with the oxidation time, and finally the matted fibers were not observed (Fig. -4C). In the pentaerythritol tetraoctanoate base grease, the $\mathrm{L} / \mathrm{D}$ value and size of the mat of the fibers almost never changed with the oxidation as shown in Fig.-5. Therefore, it is likely that the oxidation product of the base oil also affects the shape and size of soap micelles in the grease.

\section{Conclusion}

The phase behavior of the grease and the shape and size of the fibers formed by the soap micelles in the grease were significantly affected by the oxidation stability of the base oil. Some properties of the grease prepared with the more stable base oil for oxidation are hardly affected by the oxidation. This may suggest that the physical and chemical properties of the base oil could not be neglected to study on the phase behavior of the grease. For the application of the grease as a lubricant, more detail studies on the properties of the grease must be made from the different point of view.

\section{Acknowledgements}

The authors wish to thank Mr. H. Kageyama of Kyodo Yushi Co., Ltd.

(Received Oct. 19, 1976)

\section{References}

1) R.D. Vold and M.J. Vold, J. Colloid Sci., 5, 1 (1950).

2) M.J. Vold and R.D. Vold, J. Inst. Petrol., 38, 155 (1952).

3) R.M. Suggitt, NLGI Spokesman, 24, 367(1960).

4) D.B. Cox and J.F. McGlynn, Anal. Chem., 29, 960 (1957).

5) D.B. Cox, J. Phys. Chem., 62, 1254 (1958).

6) Y. Uzu, Yukagaku, 24, 261 (1975).

7) T. Sakurai, S. Hironaka, and T. Katafuchi, NLGI Spokesman, 38, 360 (1975). 